\title{
PROYECTO DE EXTENSIÓN PARA EMPRENDEDORES
}

Marcos Lavandera $\left(^{*}\right)$

\section{RESUMEN}

Desde hace más de seis años la Facultad de Ingeniería, principalmente a través del Departamento de Ingeniería Industrial, está trabajando sistemáticamente en el fomento de la cultura emprendedo$\mathrm{ra}$, realizando diversas actividades de capacitación y asistencia técnica abiertas a la comunidad, varias de ellas en conjunto con las organizaciones Co-partícipes.

Este proyecto pretende formalizar y sistematizar un grupo de trabajo interinstitucional que albergue todas estas actividades a la vez que organice otras en el mismo sentido. La existencia de un proyecto específico es orientadora y motivadora de acciones que cubran la necesidad de seguir trabajando metodológicamente para la generación de un ambiente emprendedor en la FIO y colaborar, con emprendedores de la comunidad no universitaria, en un mutuo intercambio de saberes. A través de este proyecto se establecen lazos de trabajo con diversas instituciones, promoviendo una sinergia en la construcción de redes que tendrá impacto directo sobre los emprendedores y el desarrollo de empresas sustentables.
Además, existen programas de financiamiento para emprendedores, pero existen falencias en el acompañamiento previo (elaboración del proyecto) y posterior (puesta en marcha y gestión). Este diagnóstico es compartido por la Agencia de Desarrollo Local de la Municipalidad de Olavarría (ADELO) quien se suma a este proyecto para aportar su experiencia y compartir el trabajo para los emprendedores. En el mismo sentido las demás organizaciones co-participantes aportan metodologías y sus propios programas para optimizar el abordaje de la temática, potenciando los resultados que cada uno prevé.

\section{PALABRAS CLAVE:}

Emprendedorismo - Equidad social Cultura Emprendedora - Economía Social - Ingeniería

\section{INTRODUCCIÓN}

En los últimos años el contexto macroeconómico abrió nuevas oportunidades para la generación de emprendimientos, se

(*) Ingeniero Químico y Magister en Administración de Negocios. Secretario de Extensión, Vinculación y Transferencia de la Facultad de Ingeniería de Olavarría. Jefe de Trabajos Prácticos Ordinario, Dpto. de Ing. Industrial. Integrante del Consejo Directivo de la Cátedra Abierta Latinoamericana de Innovación y Emprendedorismo (CALIYE). Director del Proyecto de Extensión "Para Emprendedores" y Director del Centro de Desarrollo Emprendedor e Innovación (CDEI) de la Facultad de Ingeniería de Olavarría. Universidad Nacional del Centro de la Provincia de Buenos Aires (UNICEN).Argentina. E-mail: marcos@fio.unicen.edu.ar 
produce un quiebre en el modelo económico de acumulación. Se inician una serie de cambios estructurales a nivel político y económico que modifican el eje de discusión en la agenda pública: de la política social de carácter reparador de la pobreza a la discusión de la redistribución de la renta. En este sentido, los emprendimientos productivos encuentran las condiciones propicias para su desarrollo, creando valor agregado en origen, articulando vertical y horizontalmente, consolidando la industria nacional.

Si bien es mucho lo que se ha avanzado, en general, podemos observar que existe un déficit de los programas de apoyo a los emprendedores, y es que, a nuestro entender, se agotan en la capacitación y el mejoramiento de habilidades, sin poder visualizar que esto es parte de una solución que requiere de múltiples dispositivos para poder abordar la complejidad de esta problemática. De acuerdo a las experiencias compartidas, el problema se agrava cuando el emprendedor debe decidir en soledad, sin poder efectuar lecturas de los contextos, de su propia organización, de su sector productivo y fundamentalmente cuando no puede aprovechar las oportunidades y evitar o transformar las amenazas para el éxito de su empresa. La opinión externa con otros conocimientos puede facilitar la mejora en las decisiones, sin embargo las empresas nacientes en general no pueden acceder a la contratación de servicios profesionales que puedan comprender la problemática socio económica por la que atraviesan los emprendimientos productivos de la economía social; situación que contribuye al aumento de la tasa de mortalidad de estas unidades productivas.

El proyecto apunta a realizar aportes para atender estos problemas, el del asesoramiento y seguimiento, primero en pequeña escala y dejar las bases para una futura incubadora de empresas, que deberá ser parte de la oferta permanente de nuestra Universidad Nacional a la sociedad/región.

Las pequeñas empresas, emprendimientos unipersonales y de la economía social, en nuestro país, siguen constituyendo, en términos absolutos, el sector de la economía de mayor flexibilidad y con mayor capacidad real y/o potencial de absorción de mano de obra. Es un sector que la Universidad debe conocer y del cual aprender y transferir, a través de estudiantes y docentes, conocimientos que colaboren con su desarrollo. Se contribuye también con la cultura emprendedora en la formación profesional de los futuros ingenieros.

Respecto al rol de la Universidad, Laukkanen $(2000)^{[1]}$ considera al hecho de formar emprendedores, como la "tercera obligación" de las universidades, actuando como motor de desarrollo y de transferencia de la tecnología. Asimismo Gibb (2002) ${ }^{[2]}$ considera que la formación de emprendedores es fundamental para la sociedad en su conjunto, esfuerzo que debería de abarcar a toda la Universidad.

Es en el contexto del estudio del comportamiento emprendedor donde surge

${ }^{[1]}$ LIÑÁN, F. and Chen, Y.-W. 2006. "Testing the entre preneurial intention model on a two - country sample". Universitat Autonoma de Barcelona. Departament d Economia del Empresa. Working Paper 06/07.

${ }^{[2]} \mathrm{GIBB}, \mathrm{A}$. ( 2002). "In pursuit of a new entreprise and entrepreneurship paradigm for learning: creative destruction, new values, new ways of doing things and new combination of knowledge". International Journal of Management Reviews. 4(3):233- 269. 
de Liñan (2006) ${ }^{[3]}$ el concepto de "clima de emprendimiento" en las universidades, que plantea la hipótesis de que "el clima, impacta en la intención emprendedora de sus estudiantes". Se concluye que "la experiencia y la forma de enseñanza, incide en la intención, por lo que el "clima de emprendimiento", existente en una facultad, repercute fuertemente en la intención emprendedora de sus estudiantes".

Se trabaja con otros organismos, instituciones y programas relacionados con la temática, como Proyecto Jóvenes Emprendedores Rurales del MinAGPyA de la Nación $^{[4]}{ }^{[1]}$ la Comisión de Investigaciones Científicas de la Provincia de Buenos Aires, Agencia de Desarrollo Local (Adelo) [5], Red Provincial de Formadores para el Desarrollo del Emprendedorismo ${ }^{[6]}$, Proyecto "Acompañando Emprendedores" ( Facultad de Ciencias Económicas y Sociales de la Universidad Nacional de Mar del Plata ${ }^{[7]}$, la Defensoría Pública del Dpto. Judicial de Azul, la ONG Caminos (administradora de los Banquitos Populares de la Buena Fe, del Ministerio de Desarrollo Social de la Nación), la Fundación "Redes de Conocimiento" ${ }^{[8]}$, la Federación Bonaerense de Parques Científicos y Tecnológi$\cos ^{[9]} \mathrm{e}$ incubadoras de otras universidades.

Se espera entonces, intensificar el apoyo a emprendedores y que los estudiantes, docentes y no docentes de la FIO transfieran sus conocimientos a la vez que toman contacto con una realidad social, productiva y económica con la cual tiene escaso contacto. Los emprendedores se verán beneficiados con este proyecto al integrarse a un espacio interdisciplinario e interinstitucional sinérgico para la atención de sus necesidades y concreción de proyectos.

\section{FUNDAMENTACIÓN}

Es evidente que el crecimiento económico no es igual a desarrollo y distribución del ingreso, y a su vez, la distribución del ingreso no necesariamente implica la creación de trabajo digno. Las experiencias de la economía social tienden a la reproducción ampliada de la vida en condiciones de igualdad y reciprocidad, orientando a la organización socio-productiva como un proyecto colectivo. Es necesario avanzar en la construcción de esta otra economía, fundada en valores y principios solidarios, coherentes con el nuevo paradigma inclusivo, al supeditar la riqueza económica al ser humano y al trabajo, privilegiando la construcción de un patrimonio colectivo. En este contexto la Universidad no puede estar ausente. Se debe recrear la tríada Universidad - Sociedad Civil - Estado para recuperar las capacidades industriales y productivas, siendo la colaboración entre estos sectores una herramienta importante para construir un sólido entramado económico social con aptitudes para competir, innovar y ser sustentables. El proyecto apunta a generar una red sectorial que agrupe a

\footnotetext{
${ }^{[3]}$ LAUKKANEN, M. (2000) "Exploring alternative approaches in high-level entrepreneurship education: creating micromechanisms for endogenous regional growth" Entrepreneurship \& Regional development. 12 Jan-Mar.p25

${ }^{[4]}$ http://www.jovenesrurales.gob.ar/

${ }^{[5]}$ http://www.olavarria.gov.ar/adelo/

${ }^{[6]} \mathrm{http}: / /$ www.redemprendedores.org.ar/

${ }^{[7]}$ https://www.facebook.com/acompanandoemprendedores.fceysunmdp?fref=ts

${ }^{[8]}$ https://www.facebook.com/FundacionRedesDelConocimiento

${ }^{[9]}$ http://www.parquescyt.org.ar/
} 
los emprendedores, para que con la correcta formulación del Proyecto a emprender y una adecuada información mejorar las posibilidades comerciales, tecnológicas, de redes, acceso a los recursos y gestión.

La Facultad de Ingeniería dispone de los recursos humanos necesarios para llevar adelante el proyecto tanto de manera directa como a través de las redes institucionales que ha integrado en este tiempo y que se suman como co-partícipes, aportando también recursos humanos, material didáctico, programas de apoyo y metodología para asistencia a emprendedores. Además la Facultad pone a disposición su infraestructura y equipos de apoyo administrativo.

Los estudiantes participantes en las actividades tienen el reconocimiento del requisito de Formación Social y Humanística de acuerdo a la normativa vigente en la currícula de las carreras $^{[10]}$ de la Facultad de Ingeniería.

\section{METODOLOGÍA UTILIZADA}

En primera instancia se acuerdan grupalmente los criterios y técnicas a utilizar, adecuadas a las características y particularidades institucionales de las organizaciones co-partícipes.

Con esto se realizan los talleres de capacitaciones en emprendedorismo para estudiantes, emprendedores, docentes y emprendedores.

Asistencias técnicas a los emprendedores. Primero se detectan las demandas y luego se realiza el proceso de tutorías con estudiantes y docentes de acuerdo a la complejidad del caso.

Herramientas metodológicas: aprendizaje vivencial - talleres - cursos - entrevistas - trabajo en campo - Exposiciones de casos - Ferias - Exposición de productos.

Si bien el abordaje es personalizado y presenciales, se dispone también de un espacio en Facebook ${ }^{[11]}$ y una página web ${ }^{[12]}$ para mejorar la comunicación con el entorno y como herramientas de gestión del proyecto.

\section{DESARROLLO}

El proyecto propone como,

\section{Objetivo General}

- Fomentar la cultura emprendedora a través de un espacio colaborativo, interdisciplinario e interinstitucional, dentro del ámbito universitario.

\section{Objetivos Específicos}

- Fortalecer la red interinstitucional en relación a la temática.

- Formar a los integrantes del proyecto como facilitadores - tutores de emprendimientos.

- Acercar a los emprendedores a los servicios y conocimientos profesionales.

- Facilitarle a los emprendedores el acceso a información relevante para su desarrollo y el del emprendimiento.

\footnotetext{
${ }^{[10]}$ Res. C. A. Fac. Ing. No 101/04. Actividades de Formación Social y Humanística

${ }^{[11]} \mathrm{https} / / /$ www.facebook.com/pepe.fio?fref=tl_fr_box\&pnref=lhc.friends

${ }^{[12]} \mathrm{http}: / /$ pepe.fio.unicen.edu.ar/
} 
- Capacitar a emprendedores en las distintas áreas y momentos del proceso emprendedor.

- Desarrollar capacidades de innovación, asociativas y de trabajo en red entre los emprendedores.

- Contribuir a la formación en competencias emprendedoras de los estudiantes.

- Integrar las funciones de docencia, investigación y extensión en torno a la temática emprendedora.

- Motivar la retroalimentación permanente con Graduados que llevan adelante sus proyectos o empresas y el acercamiento y vinculación de los mismos con los estudiantes de la Facultad de Ingeniería.

- Contribuir a la formación de una masa crítica de actores universitarios y no universitarios, de manera coordinada y metodológica, para el apoyo a emprendedores.

- Elaborar una base de datos de emprendedores.

- Contar con una base de datos de programas de apoyo a emprendedores.

Organizaciones Co-partícipes

- Agencia de Desarrollo Local de Olavarría (ADELO).

- Red Provincial de Formadores para el Desarrollo del Emprendedorismo (Comisión de Investigaciones Científicas de la Provincia de Buenos Aires).

- Proyecto Jóvenes Emprendedores
Rurales (Ministerio de Agricultura, Ganadería y Pesca de la Nación)

- Centro de Innovación y Creación de Empresas (CICE) - UNCPBA ${ }^{[13]}$.

- Proyecto "Acompañando Emprendedores", de la Facultad de Ciencias Económicas y Sociales de la Universidad $\mathrm{Na-}$ cional de Mar del Plata.

- Defensoría Pública del Departamento Judicial de Azul.

- Centro de Referencia Azul de la Secretaria de Niñez y Adolescencia de la provincia de Buenos Aires.

- Programa para el Abordaje de Problemáticas Sociales y Relaciones con la $\mathrm{Co}_{-}$ munidad - Defensa Pública Departamento Judicial de Azul.

- Centro de Referencia del Ministerio de Desarrollo Social de la Nación.

- Federación de Tierras, viviendas y hábitat de la Rep.Argentina.Del.Olavarría.

- Asociación Civil Caminos.

- Organizaciones vinculadas a la discapacidad.

- Escuela de Educación Agropecuaria de Olavarría

\section{Destinatarios}

- Estudiantes y docentes de la Facultad de Ingeniería de Olavarría - UNICEN

- Emprendedores: Personas con ideas, proyectos o emprendimientos en marcha que requieran apoyo para llevarlos adelante,

${ }^{[13]}$ http://www.cice.unicen.edu.ar/ 
asistencia técnica y/o capacitación

- Jóvenes en Contexto de encierro o liberados: Jóvenes penados por la ley que buscan insertarse en la sociedad y en el mercado laboral, mediante el emprendedorismo.

- Jóvenes con Capacidades diferentes: personas que poseen discapacidad, que pertenecen a instituciones de educación especial y tienen una idea o proyecto para emprender.

- Comunidad en general.

\section{Actividades}

Durante la ejecución del proyecto se han concretado las siguientes actividades:

- Reunión de coordinación metodológica y de actividades.

- Jornada interna de sensibilización sobre emprendedorismo.

- Capacitación para Formación de Formadores en emprendedorismo (20 a 30 hs).

- Capacitación para estudiantes facilitadores de emprendimientos (20 a $30 \mathrm{hs}$ ).

- Vinculación con emprendedores.

- Tutorías, a cargo de los estudiantes voluntarios. Se realizan tutorías de $20 \mathrm{hs}$ con cada emprendimiento. Las mismas son individuales con el emprendedor y cuando se lo considere necesario, son grupales.
- Capacitación para emprendedores, a cargo de docentes y estudiantes voluntarios (30 hs).

- Conferencias y talleres sobre la temática emprendedora.

- Participación en eventos relacionados a la temática emprendedora.

- Se realizan reuniones periódicas de seguimiento y ajustes al proyecto, estas actividades se desarrollarán dentro del equipo de trabajo, con los emprendedores y, con el equipo y los emprendedores, todo según se evalúe oportunamente.

- Eventos de Innovación Abierta (24hs de Innovación ${ }^{[14]}$ - Rally Latinoamericano de Innovación ${ }^{[15]}$ - "24 hs de Innovación FIO"[16])

- Participación en convocatorias de financiamiento de proyectos.

\section{PRINCIPALES RESULTADOS}

Con la metodología utilizada y las actividades que consecuentemente se realizan se pueden mostrar resultados cuantitativos y cualitativos:

- El Proyecto fue presentado a la Primer Convocatoria de Proyectos de Extensión de la UNCPBA ${ }^{[17]}$ y aprobado por resolución de Consejo Superior No 2200 de noviembre de 2012. Y luego fue presentado a la Tercera Convocatoria de Proyectos de Extensión de la UNCPBA y aprobado por resolución de Rectorado No 116 de marzo de 2015. También ha sido aprobado y fi-

\footnotetext{
${ }^{[14]}$ http://24h-innovation.org/es/

${ }^{[15]} \mathrm{http}: / /$ www.rallydeinnovacion.org/

${ }^{[16]} \mathrm{http} / /$ pepe.fio.unicen.edu.ar/index.php/novedades/9-recientes/16-cronograma-24hs-innovacion-fio

${ }^{[17]} \mathrm{http}: / /$ extension.unicen.edu.ar/blog/?cat=427
} 
nanciado por el Programa de Voluntariado Nacional de la Secretaría de Políticas Universitarias - MEN ${ }^{[18]}$.

- Dictado del Curso CULTURA EMPRENDEDORA 2013. Asistentes: 25 Alumnos, 2 No docentes, 1 Docente y 19 Externos (estudiantes secundarios y emprendedores). En 2014, Asistentes: 31 Alumnos y 11 Externos (emprendedores)

- En los eventos de Innovación Abierta, 24 hs de INNOVACION 2013: Participaron 8 alumnos, 1 Docente, 1 Graduado, 3 Docentes Auxiliares y en 2014 (sede Olavarría): Participaron 45 alumnos, 30 alumnos externos, 5 Docentes Secundario, 9 Auxiliares (coordinación y organización). En el Rally Latinoamericano de Innovación 2014: Participaron 58 alumnos de la UNCPBA, FASTA y UNMdP. (Universidad Nacional de Mar del Plata).

\section{4:}

- Otros datos cuantitativos 2013 -

- Cantidad de reuniones realizadas: 23

- Cantidad de estudiantes participantes: 30

- Cantidad de emprendimientos asistidos: 35

- Cantidad de docentes participantes: 5

- Cantidad de instituciones co-participes: 13 $\mathrm{Hs}$

- Horas de consultoría aplicadas: +700

- Horas de capacitación: + $250 \mathrm{Hs}$

Para mejorar la comunicación y gestión del proyecto se dispone de: www.pepe.fio. unicen.edu.ar, facebook: Pepe Fio y pepe@ fio.unicen.edu.ar

A lo largo del desarrollo del proyecto, se aprecia un crecimiento sostenido en cuanto a la cantidad de emprendedores contactados, al número de estudiantes involucrados y la cantidad de actividades concretadas.

También se va afianzando la interacción e integración entre las diferentes instituciones, logrando una mejor articulación de trabajo en equipo. Cabe destacar que las instituciones que se acercan al proyecto para ampliar las propuestas y demandas de trabajo en conjunto, comprometen trabajo efectivo para concretar los objetivos que se proponen en cada caso, de esta manera se logra incluirlas al funcionamiento universitario, a la vez que la universidad se involucra en las distintas realidades socio-productivas.

En las diferentes tutorías, se puede observar que el vínculo emprendedor - estudiantes, actúa como Modelo de Rol positivo en ambos sentidos. Los estudiantes que participan de las actividades de tutorías o de los cursos dictados, expresan que ven más factible la idea de tener un emprendimiento propio, o de asociarse para realizarlo y que se sienten más preparados para afrontar dicho desafío. Las competencias emprendedoras evidentemente se incorporan en los estudiantes en el hacer, con las vivencias.

Paulatinamente comienza a generarse un mejor "clima de emprendimiento" en la Facultad, muestra de ello es la reciente creación del Centro de Desarrollo Emprendedor e Innovación (CDEI) ${ }^{19]}$, cuyo Objetivo General es: "Fomentar la cultura emprendedora y la innovación, facilitando la generación de nuevos emprendimientos

${ }^{[18]}$ http://portales.educacion.gov.ar/spu/voluntariado-universitario/

${ }^{[19]}$ Res. C. A. Fac. Ing. No 051/15. Creación “Centro de Desarrollo Emprendedor e Innovación”. 
y fortaleciendo los ya existentes, a través de un espacio colaborativo, interdisciplinario e interinstitucional, dentro del ámbito universitario, para el apoyo a emprendedores que contribuyan al desarrollo económico regional con igualdad de oportunidades y equidad social, integrando la extensión, la investigación y la docencia" y responde al siguiente Modelo Conceptual:

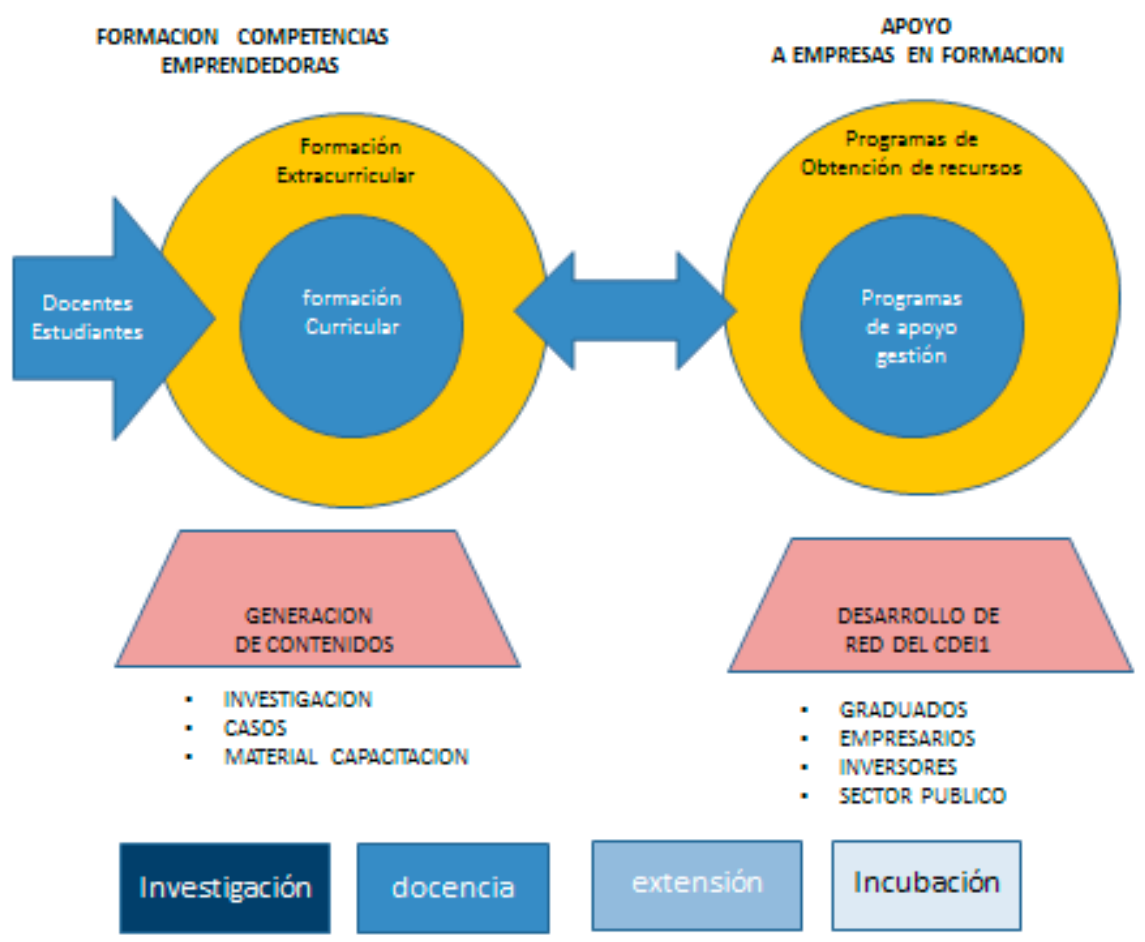

\section{DISCUSIÓN Y CONCLUSIO-} NES

De esta manera el Proyecto de Extensión "Para Emprendedores", con la vital participación estudiantil ha tenido un muy alto impacto institucional comenzando el camino de la integralidad (docencia - extensión - investigación).

Es una experiencia concreta en la que se ponen en juego distintas actividades de aprendizaje vivencial, una de las alternativas didácticas basadas en el ciclo de $\mathrm{Kolb}^{[20]}$. Se confirma de esta manera que las competen- cias emprendedoras y el espíritu emprendedor se aprende en la acción. Es importante también destacar el posicionamiento ideológico de sobre "el emprender", como el conjunto de acciones que conducen a un objetivo, más allá de la creación de empresas, deseable por cierto pero no excluyente de la concreción de otros emprendimientos no empresariales y que contribuyen mucho a la formación de los estudiantes.

En este sentido, en las actividades de innovación abierta hemos encontrado un espacio muy rico para adquirir competencias y generar ideas para luego con-

${ }^{[20]}$ Kolb, A. and Kolb, D.A. (2001) Experiential Learning Theory Bibliography 1971/2001, Boston, McBer and Co 
tinuarlas como oportunidades o proyectos. Logramos así avanzar teórica y prácticamente en el proceso emprendedor.

La intensa relación con el contexto en sus diversas formas y con distintos actores es sin dudas una herramienta trascendental en la formación de los estudiantes, estar con actividades concretas fuera de la Facultad resulta de alto impacto en su visión del mundo y el entorno en el cual les tocará actuar.

En cuanto a la motivación del espíritu empresarial, la cercanía con emprendedores de distintos rubros, el involucrarse con estilos de vida muy diferentes, además de actividades como el "Living Emprendedor", genera un modelo de rol muy motivador, a la vez de un espacio de aprendizaje compartido muy intenso, ya que los estudiantes también contribuyen con sus conocimientos técnicos al desarrollo de los emprendimientos.

Los docentes se siguen incorporando al grupo, formal o informalmente, generando un intercambio didáctico y humano por demás interesante, también un rico vínculo con los estudiantes que nos permite repensar las formas de "enseñar".

Este proyecto es una invitación al debate sobre la integralidad en la universidad y sus distintas formas de abordaje, es una invitación a "romper" con los esquemas tradicionales de relacionamiento con los estudiantes, al uso de la informalidad formal como forma de generar y compartir conocimientos. Es una forma más de poner la universidad en la calle y la calle en la universidad, en una temática, el Emprendedorismo, que contribuye al desarrollo de todos los sectores y actores que participan.

Como dice nuestro slogan: "Si tenés ganas de aprender a emprender y de emprender para aprender, hacete amigo del PEPE”.

Los resultados obtenidos demuestran que el modelo conceptual que estamos iniciando es viable, las actividades curriculares acompañadas de otras no curriculares y principalmente el aprendizaje vivencial, contribuyen a la formación en competencias emprendedoras. Sobre este modelo nos falta aún avanzar en los aspectos de investigación, desarrollo de materiales didácticos propios y también, con el tiempo, lograr sistematizar el proceso de incubación de emprendimientos.

Esta experiencia realizada en una facultad de ingeniería, sin dudas es replicable en cualquier otra área, de hecho nos falta incrementar la interdisciplina. Varias de las experiencias recogidas como antecedentes provienen de otras áreas. Este proyecto no tiene un carácter disciplinar marcado, es muy flexible a otros conocimientos, ciencias sociales, por ejemplo. 